NASA

Technical Memorandum 101378

AVSCOM

Technical Report 88-C-023

\title{
An Expert System for Restructurable Control
}

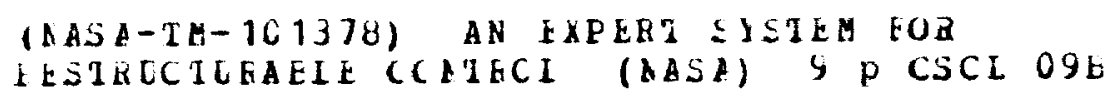

No9-12309

Unclas

G3/63 0174590

Jonathan Litt

Propulsion Directorate

U.S. Army Aviation Research and Technology Activity-AVSCOM

Lewis Research Center

Cleveland, Ohio

Prepared for

Space Operations Automation and Robotics (SOAR 88)

cosponsored by the U.S. Air Force, National Aeronautics and Space

Administration, and Wright State University

Dayton, Ohio, July 20-23, 1988

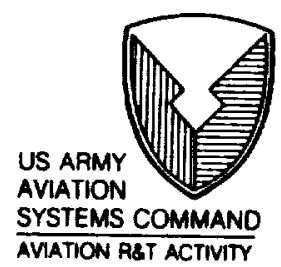


-

‘

. 


\title{
AN EXPERT SYSTEM FOR RISTRUCTURABLE CONTROL
}

\author{
Jonathari Litt \\ Propulsion Directorate \\ U.S. Army Aviation Research and Technology Activity - AVSCOM \\ Lewis Research Center \\ Cleveland, Ohio 44135
}

\begin{abstract}
SUMMFIRY
Work in progress on an expert systell which restructures and tunes control systems on-line is presented. The exper: system coordinates the different methods for redesigning and implementing the control strategies due to system changes. The research is directed toward aircraft and jet engine applications. The implementation is written in LISP and is currently running on a special purpose LISP machine.
\end{abstract}

\section{INTRODUCTION}

A restructurable control system has the ability to redesign itself on-line to compensate for a significant change in the system. Restructurability is important to mission effectiveness because it allows a closed-loop system to continue operating in an acceptable manner even after major changes to the system. Here the closed-loop system consists of a controller and plant. Examples of systems with major changes are aircrat with battle damage or engines with foreign object damage. With an invariant control system designed for the nominal plant, an aircraft that experienced battle damage may now only be able to limp home. In the worst case it would be unstable. With a redesigned control system for the new, altered plant, the plane is more likely to return safely and it may be able to carry out all or part of its mission with only slightly reduced capabilities.

Restructurable control is applicable to systems which experience mechanical problems such as actuator or control surface fallures and where the capability lost due to fallure is wholly or partially available in some other component or components. Most of the redesign strategies in the literature work by redistributing the forces and moments of the failed actuators or missing surfaces over the remaining redundant components to compensate for the lost components. The methods differ in the redesign approach they employ. The research by Looze et al. has concent ated on a linear quadratic approach to the redesign procedure (ref. 1). Horowitz et al. have applied quantative feedback theory to control system reconfiguration (ref. 2). Raza and Silverthorn have used the pseudoinverse of the control matrix and generalized input vectors to achieve the desired responses along the orthogonal axes (ref. 3). The technique in reference 3 is similar to the control mixer concept for reconfiguration described by Rattan (ref. 4).

The goal of this paper is to describe a way to tie together some of the previous work in the field so as to achieve a highly survivable control system. A highly survivable system can successfully restructure in response to a multitude of different failures. In general, previous restructurable controllers have been specifically desigied for a single failure type. 
Each design method used is valld for its speciflc application. However, none is "optimal" nor even applicable in all situations. Thus, to achieve a highly survivable system, it is necessary to identify the current dynamic characteristics of the system and to determine which of the possible solutions is the best in some sense under the given circumstances. To accomplish this decision making in an uncertain environment with potentially conflicting mission objectives, some type of intelligence will be required. Hence the concept of an expert system to coordinate the different redesign strategies is proposed.

\section{BACKGROUND}

The idea of restructurable control has appeared recently, mainly with respect to aircraft. Battle damage has been considered a perfect application for the research. Commercial airliners are also a possible vehicle for the work. Several accidents and near accidents where the pilot was able to recover and land the plane after analyzing the problem have been discussed in relation to restructurable control (ref. 5).

Thus this strategy is very attractive for both civilian and military aeronautics and propulsion applications. Creating the ability in a plane to restructure its control system after damage in order to continue at a level of performance similar to its original design specifications is highly desirable. It is also important to remember that the main ideas here are not limited to airplanes. They can be applied to a wide variety of systems with inherent redundancy.

\section{EXPERT SYSTEM COMPONENTS}

An expert system consists of three independent parts: a rule base, a knowledge base, and an inference engine. The rule base is a set of heuristics or rules-of-thumb which apply to the type of problem at hand. The knowledge base is a collection of information specific to the current situation. The inference engine is a program which applies the rules to the knowledge base in order to glean new information or to determine if an assumption is justified. When new information is asserted, it is stored in the knowledge base.

An inference engine can work with any appropriately structured knowledge base and rule base. This three part structure allows the inference engine to be application-independent while the application-dependent information resides in the knowledge base and rule base.

The proposed overall structure of the reconfiguration expert system is shown in figure 1. It consists of (1) an inference engine, (2) a control system restructuring knowledge and rule base, and (3) a controller tuning knowledge and rule base. The control system restructurer is already partially implemented. In the future we plan to incorporate an on-line controllertuning expert system into the overall system. It will share the inference engine with the reconfiguration expert system.

The inference engine developed for this application is capable of performing symbolic and numerical calculations required to evaluate certain rules. It can also execute generalized rules with previously established facts from 
the knowledge base to infer new facts. In addition, it has the ability to perform what-if type reasoning by trying different scenarios if more than one is appropriate.

The knowledge base of the restructurable control system consists of information about the plant and its controller. For a linear system such parameters as the system matrices and the original controller gains are stored. There are also specifications on the actuators such as linear ranges and nonlinear characteristics. Information stored here can change in response to plant changes. It is changed or updated as new facts become available.

The rule base of the control system restructurer contains rules about control system design. These range from top-level control design methods to low-level details such as definitions of controllability and observability. The rules may contain numerical expressions to be evaluated (such as whether a realization is minimal) and may contain variables to be given values by the inference engine during the discovery of new facts.

A separate knowledge base will be required for the tuning system. Following the approach of reference 6 , it will contain response characteristics assoclated with a well-tuned loop of the type in question. It also will have data on any previous responses obtained in the tuning process.

A rule base for controller tuning $w^{2} 11$ be created also. The heuristics will use the results from prevlous tuning efforts and other plant information for the next tuning attempt.

Figure 2 shows the Interaction of tlie expert system with the overall system. A significant change in the identified model of the plant will cause the expert system to restructure the control er to compensate for the alteration. After the new controller is implemented, the expert system will adjust the controller parameters to optimize the perfolmance of the closed loop system.

\section{SYSTEM CAPABILITIES}

Figure 3 shows the anticipated future setup of the overall system. It shows a hierarchy with an expert system receiving information from a system identifier and a pattern extractor. This information is used in the restructuring and tuning of the controller for whe altered plant. In the current setup, the plant simulation, the controlier, and the expert system are all written in compiled LISP running on an LHI Lambda LISP machine. The system identifier and the pattern extractor are not yet implemented. The simulation consists of a realization of a linearized system in the form of matrices $(A, B, C, D)$ and the state is evolved using Euler integration. Presently the expert system uses a model of the plant directly from the simulation. The linear model is of the form:

$$
\begin{aligned}
& \dot{x}=A x+B u \\
& y=C x+D u
\end{aligned}
$$

A change in the model prompts the expert system to analyze and redesign the control. The new controller replaces tha old one in the simulation and the state continues to evolve. 
The restructuring strategies that the expert system can currently use involve the pseudoinverse of $B$ (refs. 3 and 4 ). The expert system takes a realization $(A, B, C)$ and manipulates it, using the Kalman Structure Theorem for instance, until it is minimal and $B^{T} B$ has full rank. If the expert system can achieve this goal, the equation

$$
K=\left(B^{\top} B\right)^{-1} B^{\top}\left[A-\left(A_{0}-B_{0} K_{0}\right)\right]
$$

is used to determine the new controller matrix. Here $A$ and $B$ are the altered system matrices and $\left(A_{0}-B_{0} K_{0}\right)$ is the reduced order version of the original closed-loop system matrix of the full order model.

Examples of the heuristics used in the situation described above are:

(1) If $(A, B, C)$ is controllable and observable then realization is minimal.

(2) If $B^{T} B$ is full rank then pseudoinverse of $B$ exists.

(3) If $(A, B, C)$ is not minimal and $(A, B, C)$ is minimum phase then find a minimal realization.

(4) If pseudoinverse of $B$ exists and realization is minimal

then

$$
K=\left(B^{\top} B\right)^{-1} B^{\top}\left[A-\left(A_{0}-B_{0} K_{0}\right)\right]
$$

These rules are typical of the heuristics contained in the rule base.

A user interface exists for use in the development stage. In a delivery system there will be no need for such an environment as the system will run without human intervention.

The expert system executes only when invoked, for example when the control needs to be redesigned. Currently, it is invoked by manually halting the simulation and typing the command to execute the expert system. The simulation must then be restarted. This is necessary at present because the simulation and the expert system both run on the same processor and no system identification scheme has yet been implemented. In the future the identifier will communicate with the expert system and cause it to start redesigning when a significant change in the system matrices occurs.

\section{CONCLUDING REMARKS}

The expert system is able to handle a variety of reconfiguration situations. For these cases, the new controller is designed and implemented in a matter of seconds. Naturally the redesign time depends on the order of the system. 
At present a few of the control design algorithms from the literature have been implemented. More have to be includad in addition to incorporating any other work, both new and existing, that is deemed necessary for the system to work well.

Some work has been done in the area of controller tuning by pattern recognition techniques for single-input single-output systems (ref. 6 ). We intend to extend the methodology to multiple-inpat-multiple-output systems.

Currently the LISP machine performs the numerical calculations. For real-time execution of the system, a special purpose numeric processor, such as an array processor, will be required.

A system identifier will be implemented in the future. In the near term one might be implemented on the LISP machine. Eventually a microprocessorbased system identifier should be connected to the plant and signal the expert system if a significant change occurs in the model.

An on-line pattern extractor which will determine the response features will also have to be developed. These features will be passed to the knowledge base of the tuning expert system.

The simulation currently residing within the Lambda will be moved to an Applied Dynamics ADI00 simulation computer. This will allow a nonlinear. real-time simulation to be implemented. When the interface between the two is completed, the capability will exist to test the expert system in a realistic situation.

\section{REFEREMCES}

1. Looze, D.P., et al.: An Automatic Redesign Approach for Restructurable Control Systems. Proceedings of the IEEE National Aerospace and Electronics Conference, NAECON 1985, Vol. 1, IEEE, 1985, pp. 570-577.

2. Horowitz, I.; Arnold, P.B.; and Houpis, C.H.: YFl6CCV Flight Control System Reconfiguration Design Using Quantitative Feedback Theory. Proceedings of the IEEE National Aerospace and Electronics Conference, NAECON 1985, Vol. 1, IEEE, 1985, pp. 578-585.

3. Raza, S.J.; and Silverthorn, J.T.: Use of the Pseudo-Inverse for Design of a Reconfigurable Flight Control System. AIAA Guidance, Navigation and Control Conference, AIAA, 1985, pp. 349-356.

4. Rattan, K.S.: Evaluation of Control Mixer Concept for Reconfiguration of Flight Control System. Proceedings of the IEEE National Aerospace and Electronics Conference, NAECON 1985, Vol. 1, IEEE, 1985, pp. 560-569.

5. Montoya, R.J., et al., eds.: Restructurable Controls. NASA CP-2277, 1982.

6. Litt, J.: An Expert System for Adaptive PID Tuning Base on Pattern Recognition Techniques. Instrumentation in the Chemical and Petroleum Industries, vol. 18, Instrument Soclety of America, 1986, pp. 87-104. 


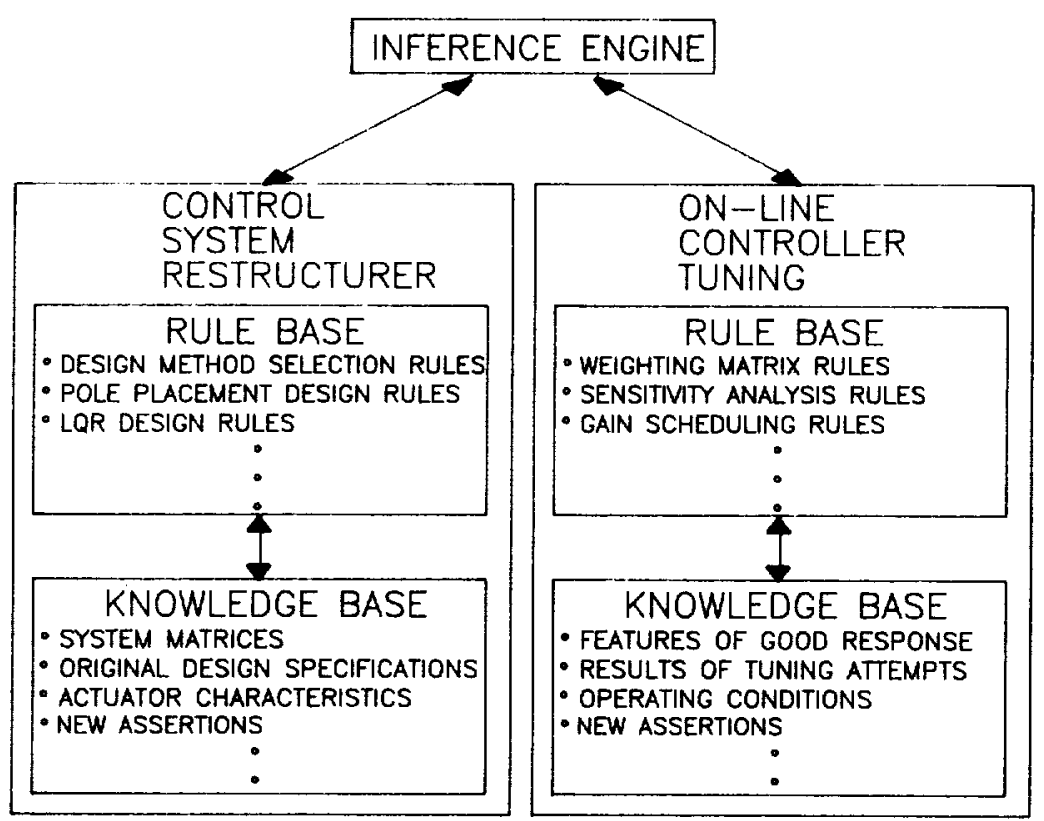

Figure 1 - Structure of the Expert Systems

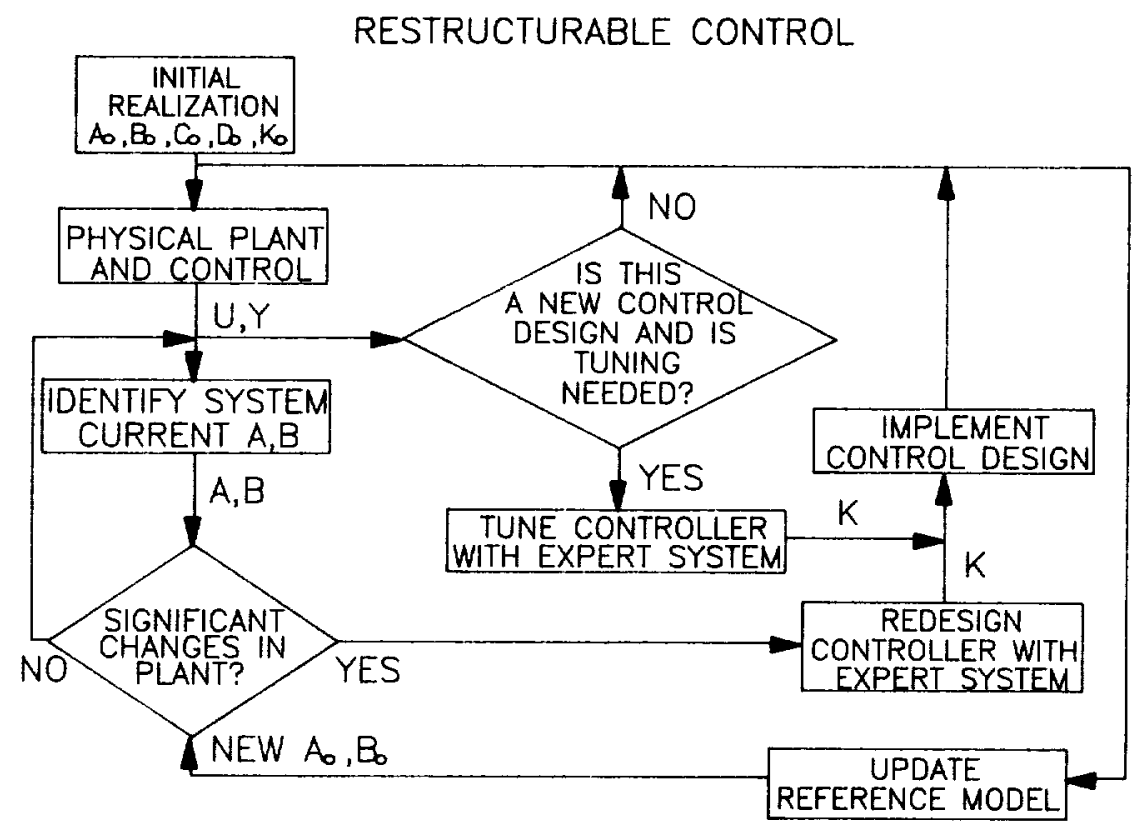

Figure 2 - Expert System Interaction with Overall System 


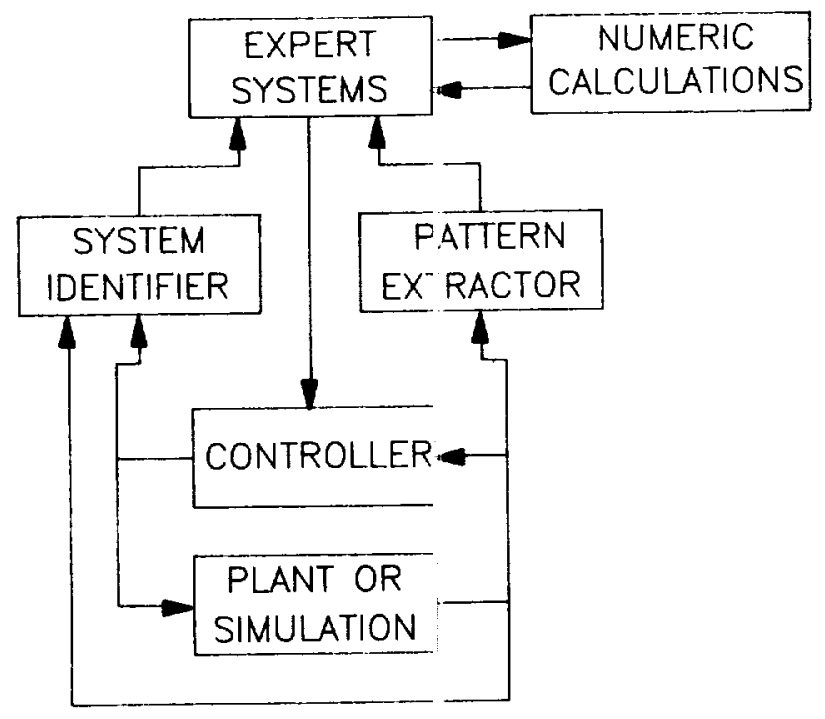

Figure 3 - Overall Hordware: Configuration 


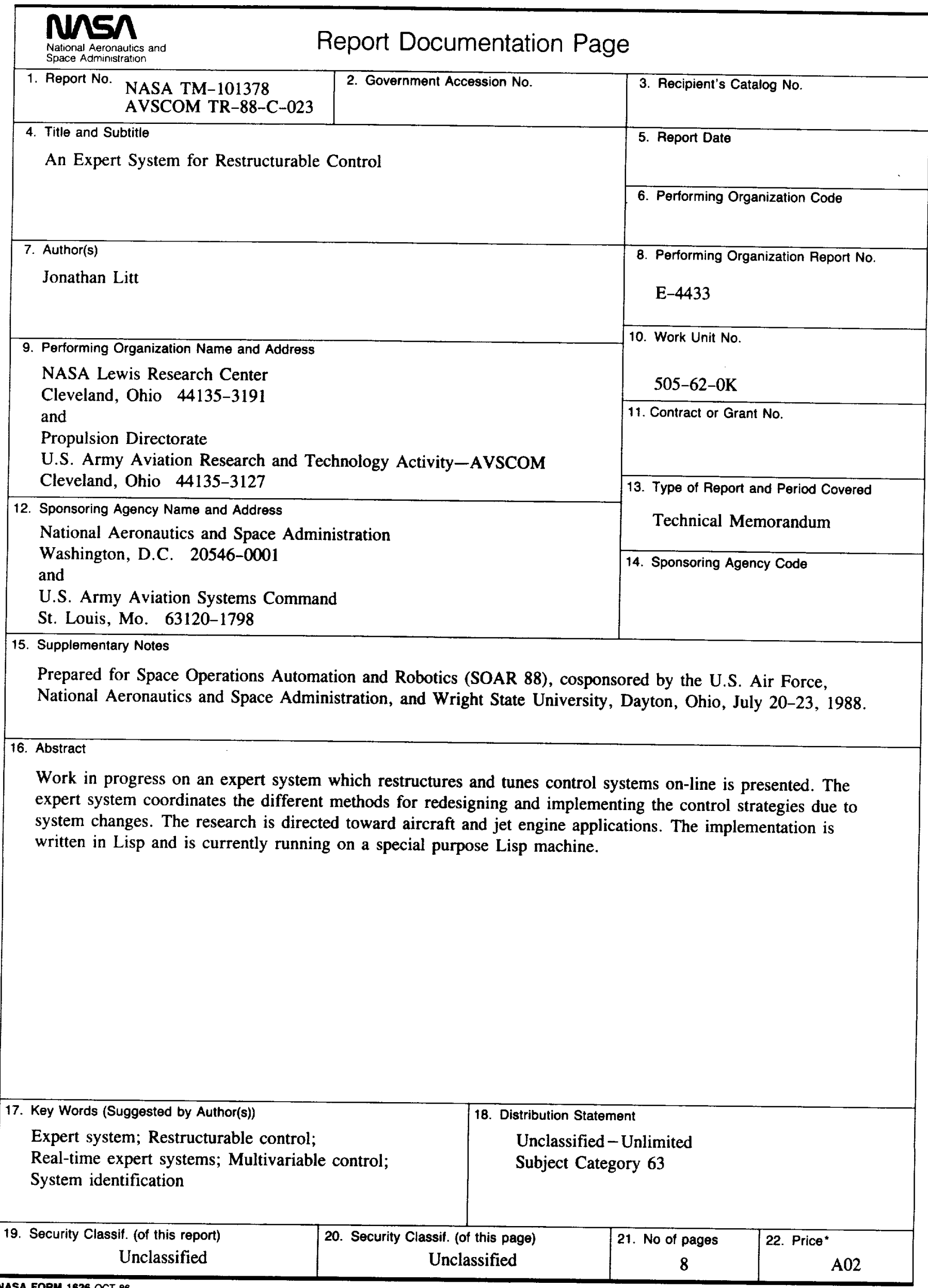

